\title{
Attention Concentration
}

National Cancer Institute

\section{Source}

National Cancer Institute. Attention Concentration. NCI Thesaurus. Code C41209.

The act or process of concentrating, focusing the attention and mental energy on a single object to the exclusion of others. 